\title{
Risk Factors for Piperacillin-Tazobactam-Resistant Pseudomonas aeruginosa among Hospitalized Patients
}

\author{
Anthony D. Harris, ${ }^{1,2 *}$ Eli Perencevich, ${ }^{3}$ Mary-Claire Roghmann, ${ }^{1,2}$ Glenn Morris, ${ }^{1}$ \\ Keith S. Kaye, ${ }^{4}$ and Judith A. Johnson ${ }^{2,5}$ \\ Department of Epidemiology and Preventive Medicine ${ }^{1}$ and Department of Pathology, ${ }^{5}$ University of Maryland, and VA Maryland \\ Health Care System, ${ }^{2}$ Baltimore, Maryland; Beth Israel Deaconess Medical Center, Boston, Massachusetts ${ }^{3}$; and Division of \\ Infectious Diseases and International Health, Duke University Medical Center, Durham, North Carolina ${ }^{4}$
}

Received 5 June 2001/Returned for modification 29 September 2001/Accepted 7 December 2001

\begin{abstract}
Antimicrobial resistance is an emerging problem with Pseudomonas aeruginosa. This study determined risk factors for the recovery of piperacillin-tazobactam-resistant $P$. aeruginosa from clinical cultures from hospitalized patients. A case-control study design was used to compare two groups of case patients with control patients. The first group of case patients was defined by nosocomial isolation of piperacillin-tazobactamresistant $\boldsymbol{P}$. aeruginosa, and the second group of cases yielded piperacillin-tazobactam-susceptible $P$. aeruginosa. Controls were selected in a 6:1 ratio from the same medical or surgical services among which piperacillintazobactam-resistant $P$. aeruginosa arose in patients. Risk factors analyzed included antimicrobial drug exposure, comorbid conditions, and demographics. Bivariate and multivariable analyses were performed. Piperacillin-tazobactam-resistant $P$. aeruginosa was isolated from 179 patients, and piperacillin-tazobactamsusceptible $P$. aeruginosa was isolated from 624 patients over a 2.5 -year period. Piperacillin-tazobactam (odds ratio $[\mathrm{OR}]=6.82 ; 95 \%$ confidence interval $[\mathrm{CI}], 4.56$ to 10.21$)$, imipenem $(\mathrm{OR}=2.42 ; 95 \% \mathrm{CI}, 1.19$ to 4.94$)$, aminoglycosides $(\mathrm{OR}=2.18 ; 95 \% \mathrm{CI}, 1.44$ to 3.28$)$, vancomycin $(\mathrm{OR}=1.87 ; 95 \% \mathrm{CI}, 1.21$ to 2.89$)$, and broad-spectrum cephalosporins $(\mathrm{OR}=2.38$; $95 \% \mathrm{CI}, 1.45$ to 3.88$)$ were the antibiotics associated with the isolation of piperacillin-tazobactam-resistant $P$. aeruginosa. Exposure to vancomycin $(\mathrm{OR}=1.53 ; 95 \% \mathrm{CI}, 1.13$ to 2.06) or ampicillin-sulbactam $(\mathrm{OR}=\mathbf{2 . 2 8}$; $95 \% \mathrm{CI}, 1.62$ to 3.21$)$ was associated with recovery of piperacillintazobactam-susceptible $\boldsymbol{P}$. aeruginosa. In this study, antibiotics associated with piperacillin-tazobactam-susceptible $\boldsymbol{P}$. aeruginosa were different from antibiotics associated with piperacillin-tazobactam-resistant $P$. aeruginosa. Piperacillin-tazobactam was a strong risk factor for piperacillin-tazobactam-resistant $P$. aeruginosa. Our results suggest that the nosocomial isolation of piperacillin-tazobactam-resistant $P$. aeruginosa may be affected by multiple antibiotics.
\end{abstract}

Pseudomonas aeruginosa is a leading cause of nosocomial infections, ranking fifth among all the pathogens reported to the National Nosocomial Infection Surveillance System from January 1990 to March 1996 (1). Piperacillin-tazobactam resistance among $P$. aeruginosa strains is an emerging problem; among 127 intensive care units (ICUs) that participate in the Centers for Disease Control and Prevention's ICARE Project, 6,917 $P$. aeruginosa isolates underwent susceptibility testing, and the rate of piperacillin resistance was $14.4 \%$ (http://www .cdc.gov/ncidod/hip/NNIS/AR_Surv1198.htm). Since the chromosomal $\beta$-lactamases of $P$. aeruginosa are not effectively inhibited by tazobactam, the rate of resistance to piperacillintazobactam is likely of a magnitude similar to that for the rate of resistance to piperacillin (2).

The aim of this study was to identify risk factors for the nosocomial isolation of piperacillin-tazobactam-resistant $P$. aeruginosa from clinical specimens. Risk factors of particular interest were antimicrobial drug exposures. To our knowledge,

* Corresponding author. Mailing address: Division of Healthcare Outcomes Research, Department of Epidemiology and Preventive Medicine, University of Maryland School of Medicine-VA Maryland Health Care System, 10 North Greene St., Room 5D151, Baltimore, MD 21201. Phone: (410) 605-7000, ext. 5211. Fax: (410) 605-7914. E-mail: aharris@epi.umaryland.edu. no previous studies have been published on risk factors for piperacillin-tazobactam-resistant $P$. aeruginosa.

\section{MATERIALS AND METHODS}

Case definition, control definition, and study design. A case-case-control study design was used (7). Two retrospective case-control studies were concurrently performed at the University of Maryland Medical System. The acute-care hospital consists of a 609-bed acute care hospital that includes the R. Adams Cowley Shock Trauma Center and the Greenbaum Cancer Center. The first group of cases was defined as patients from whom $P$. aeruginosa resistant to piperacillintazobactam was nosocomially isolated in clinical cultures. The second group of cases was defined as patients from whom $P$. aeruginosa susceptible to piperacillintazobactam was nosocomially isolated, also in clinical cultures. Microbiologic identification of $P$. aeruginosa was done with Vitek GNI cards. Antimicrobial susceptibility testing over the entire period was done by disk diffusion following NCCLS guidelines. Zone sizes for both piperacillin and piperacillin-tazobactam were the following: susceptible, $>18 \mathrm{~mm}$; resistant, $<17 \mathrm{~mm}$.

The microbiology laboratory database was electronically searched to identify all clinical cultures positive for $P$. aeruginosa from patients admitted between 1 January 1998 and 1 July 2000. Patients from whom $P$. aeruginosa isolates were obtained within the first $48 \mathrm{~h}$ of admission were excluded. Isolates obtained from surveillance cultures were excluded. Control group 1 patients were selected from the same medical or surgical services that were being used for cases when piperacillin-tazobactam-resistant $P$. aeruginosa was isolated. Piperacillin-tazobactam-resistant $P$. aeruginosa was not isolated from patients in control group 1 during their hospital stay. Control group 2 was identical to control group 1 but additionally excluded patients with piperacillin-tazobactam-susceptible $P$. aeruginosa. Six controls were randomly chosen for each case. Controls were admitted in the same 2.5-year time period. Controls were randomly spread out over the 2.5-year period to try to diminish the effect of potential infection control and 
hygiene confounding variables. Patients admitted for less than $48 \mathrm{~h}$ were excluded from the control groups.

The rationale for a case-case-control study design is to identify risk factors for the isolation of piperacillin-tazobactam-resistant $P$. aeruginosa by contrasting two multivariable models: risk factors for isolation of $P$. aeruginosa resistant to piperacillin-tazobactam and risk factors for isolation of $P$. aeruginosa susceptible to piperacillin-tazobactam. Thus, one is able to identify variables specifically predictive of the outcome of interest, namely, piperacillin-tazobactam-resistant $P$. aeruginosa. The advantages of this study design over an alternate study design where patients with piperacillin-tazobactamresistant $P$. aeruginosa would be directly compared to control patients with piperacillin-tazobactam-susceptible $P$. aeruginosa have been outlined in previous publications (6, 7; A. D. Harris, M. H. Samore, and Y. Carmeli, Letter, Ann. Intern. Med. 133:159, 2000).

Risk factors analyzed. Data were collected from administrative, pharmacy, and laboratory computerized databases by means of a relational database management system. The relational database is maintained by the Information Technology Group of the University of Maryland. The pharmacy, microbiology, and medical demographics tables in the relational database have been validated against medical records for more than 400 patients admitted between October 1997 and January 2001. In addition, data for a random 10\% sample of cases and $5 \%$ of controls in this study were validated with medical charts. The positive and negative predictive values of the data are greater than $99 \%$ when compared to patients' medical charts.

Variables explored as possible risk factors included age, gender, underlying diseases or comorbid conditions, the Charlson score (the last two obtained by using coding of the International Classification of Diseases) (3), ICU stay prior to the outcome of interest, surgery prior to the outcome of interest, transfer from another hospital, number of admissions to the hospital in the prior year, length of hospital stay prior to the outcome of interest, i.e., time at risk (for cases, length of stay prior to $P$. aeruginosa isolation, and for controls, complete length of hospital stay) and treatment with antimicrobial drugs (analyzed individually and in groups; see Tables 1 and 2). For antimicrobial data, only antimicrobial drugs received within the hospital were analyzed. For the cases, treatment with antimicrobial drugs was included in the analysis only when the antimicrobial drugs were given within 14 days prior to isolation of $P$. aeruginosa (for case group 1, within 14 days of isolating piperacillin-tazobactam-resistant $P$. aeruginosa, and for case group 2, within 14 days of isolating piperacillin-tazobactam-susceptible $P$. aeruginosa). For controls, treatment with antimicrobial drugs during the 14 days prior to discharge was included. The rationale behind the choice of 14 days was to avoid analyzing antibiotics that patients had received during the initial phase of a lengthy admission. For example, if a patient had been admitted for 40 days prior to the nosocomial isolation of the resistant organism, we believed that antibiotics received early on in the admission were unlikely to be related to the isolation of resistant $P$. aeruginosa. However, because the 14-day period was arbitrarily chosen, statistical analyses were repeated with antibiotics received during the whole time at risk prior to the event, i.e., no 14-day limitation, and the results obtained were very similar; thus, for conciseness only the 14-day period data are presented in the Results section.

Statistical analysis. All statistical analyses were performed using SAS software version 7 (SAS Institute, Cary, N.C.). Bivariate analyses were performed separately for each of the variables. Odds ratios (ORs) and $95 \%$ confidence intervals (95\% CIs) were calculated for binomial variables; $P$ values were calculated by Fisher's exact test for binomial variables, by the Chi-square test for categorical variables with $>2$ subgroups, and by Student's $t$ test or the Wilcoxon rank-sum test for continuous variables. Multivariable logistic regression was performed using PROC LOGIT in SAS.

Variables with a $P$ value of $<0.1$ in the bivariate analysis were included in a logistic regression model for multivariable analysis. A forward selection process was used. Risk factors were checked for confounding and collinearity. Confounders were included in the multivariable models if covariate inclusion changed the coefficient of any statistically significant variable in the logistic regression model by $10 \%$ or greater. Collinearity was verified by Spearman correlation among covariates and by viewing changes in standard errors of multivariable models. All tests were two-tailed, and a $P$ value of $\leq 0.05$ was considered significant in the multivariable model.

\section{RESULTS}

During the 2.5-year study period, 179 patients with piperacillin-tazobactam-resistant $P$. aeruginosa (cases 1 ) and 624 patients with piperacillin-tazobactam-susceptible $P$. aeruginosa (cases 2) were identified. One thousand one hundred and thirty-six controls were used as controls for group 1. For 27 of these controls, piperacillin-tazobactam-susceptible $P$. aeruginosa was isolated during their admission, and thus, they were not included in control group 2. Hence, there were 1,109 patients in control group 2.

Piperacillin-tazobactam-resistant $P$. aeruginosa was most frequently recovered from respiratory secretions (43\%). Other sites of recovery included urine $(21 \%)$, wound cultures $(12 \%)$, and blood (4\%). Piperacillin-tazobactam-susceptible $P$. aeruginosa was also most frequently recovered from respiratory secretions (38\%). Other sites of recovery included urine $(31 \%)$, wound cultures $(12 \%)$, and blood $(5 \%)$. The medical or surgical services that were being used for patients with piperacillin-tazobactam-resistant $P$. aeruginosa on the date of positive culture were medicine $(20 \%)$, trauma ward $(34 \%)$, transplant $(20 \%)$, and cancer center $(2 \%)$. The medical or surgical services being used for patients with piperacillin-tazobactam-susceptible $P$. aeruginosa on the date of positive culture were medicine $(14 \%)$, trauma ward $(39 \%)$, transplant $(9 \%)$, and cancer center $(3 \%)$.

Results for the bivariate risk factor analysis for piperacillintazobactam-resistant $P$. aeruginosa are outlined in Table 1, and results for the bivariate risk factor analysis for piperacillintazobactam-susceptible $P$. aeruginosa are outlined in Table 2. The results of the multivariable risk factor analyses for both piperacillin-tazobactam-resistant $P$. aeruginosa and piperacillin-tazobactam-susceptible $P$. aeruginosa are outlined and contrasted in Table 3.

The multivariable logistic-regression analysis demonstrates that patients with nosocomial isolation of piperacillin-tazobactam-resistant $P$. aeruginosa were more likely to have been exposed to the following antibiotics in the 14 days prior to the date of positive culture: piperacillin-tazobactam $(\mathrm{OR}=6.82 ; 95 \% \mathrm{CI}, 4.56$ to 10.21$)$, imipenem $(\mathrm{OR}=2.42$; $95 \% \mathrm{CI}, 1.19$ to 4.94$)$, aminoglycosides ( $\mathrm{OR}=2.18 ; 95 \% \mathrm{CI}$, 1.44 to 3.28$)$, vancomycin $(\mathrm{OR}=1.87 ; 95 \% \mathrm{CI}, 1,21$ to 2.89$)$, and third-generation cephalosporins $(\mathrm{OR}=2.38 ; 95 \% \mathrm{CI}$, 1.45 to 3.88 ). The time at risk, defined as the amount of time from admission to positive culture date for cases, was a significant risk factor $(\mathrm{OR}=1.02 ; 95 \% \mathrm{CI}, 1.01$ to 1.03$)$; i.e., for each extra day in hospital, the risk of isolating piperacillin-tazobactam-resistant $P$. aeruginosa increased by $2 \%$. Having had an ICU stay prior to the event of interest (positive culture for cases) was also a risk factor $(\mathrm{OR}=2.59$; $95 \% \mathrm{CI}, 1.65$ to 4.06$)$. Being transferred from another medical institution on admission was also a risk factor $(\mathrm{OR}=$ 2.07; $95 \% \mathrm{CI}, 1.31$ to 3.27 ). The number of admissions to the hospital in the prior year was also a risk factor $(\mathrm{OR}=1.36$; $95 \%$ CI, 1.20 to 1.54 ).

Table 3 demonstrates that patients with nosocomial isolation of piperacillin-tazobactam-susceptible $P$. aeruginosa were more likely to have been exposed to the following antibiotics in the 14 days prior to the date of positive culture: vancomycin (OR $=1.53 ; 95 \% \mathrm{CI}, 1.13$ to 2.06$)$ and ampicillin-sulbactam $(\mathrm{OR}=$ 2.28; $95 \% \mathrm{CI}, 1.62$ to 3.21 ). Having had an ICU stay prior to the event of interest was a risk factor $(\mathrm{OR}=3.76$; $95 \% \mathrm{CI}, 3.00$ to 4.71$)$, as was age $(\mathrm{OR}=1.01 ; 95 \% \mathrm{CI}, 1.01$ to 1.02$)$. 
TABLE 1. Bivariate risk factors for the isolation of $P$. aeruginosa resistant to piperacillin-tazobactam ${ }^{a}$

\begin{tabular}{|c|c|c|c|c|c|}
\hline \multirow{2}{*}{ Variable } & \multicolumn{2}{|c|}{ Value or no. $(\%)$ for: } & \multirow{2}{*}{$P$ value } & \multirow{2}{*}{ OR } & \multirow{2}{*}{$95 \% \mathrm{CI}$} \\
\hline & Controls $^{b}$ & Cases $^{c}$ & & & \\
\hline \multicolumn{6}{|l|}{ Demographics and comorbidities } \\
\hline Mean age (yr) & 49.7 & 53.4 & 0.007 & 1.01 & $1.00-1.02$ \\
\hline Male gender & $448(39.4)$ & $76(42.5)$ & 0.44 & & \\
\hline AIDS & $29(2.6)$ & $7(3.9)$ & 0.30 & & \\
\hline Cardiac disease & $128(11.3)$ & $20(11.2)$ & 0.97 & & \\
\hline Diabetes & $227(20)$ & $44(24.6)$ & 0.16 & & \\
\hline Malignancy & $107(9.4)$ & $12(6.7)$ & 0.24 & & \\
\hline Cerebrovascular accident & $68(6)$ & $17(9.5)$ & 0.076 & 1.65 & $0.94-2.88$ \\
\hline Hepatic disease & $25(2.2)$ & $5(2.8)$ & 0.62 & & \\
\hline Renal disease & $27(2.4)$ & $7(3.9)$ & 0.23 & & \\
\hline Charlson comorbidity scale (mean) & 1.28 & 1.44 & 0.32 & & \\
\hline \multicolumn{6}{|l|}{ Variables related to hospitalization } \\
\hline Time at risk (days) ${ }^{d}$ & 10.2 & 22.9 & $<0.0001$ & 1.04 & $1.03-1.05$ \\
\hline ICU stay & $441(38.8)$ & $136(76)$ & $<0.0001$ & 4.98 & $3.47-7.17$ \\
\hline Surgery & $162(14.3)$ & $27(15.1)$ & 0.77 & & \\
\hline No. of admissions past year & 0.54 & 1.12 & $<0.0001$ & 1.30 & $1.18-1.43$ \\
\hline Transfer & $149(13.1)$ & $51(28.5)$ & $<0.0001$ & 2.64 & $1.83-3.81$ \\
\hline \multicolumn{6}{|l|}{ Antibiotics ${ }^{f}$} \\
\hline Imipenem & $25(2.2)$ & $26(14.5)$ & $<0.0001$ & 7.55 & $4.25-13.4$ \\
\hline Piperacillin-tazobactam & $129(11.4)$ & $94(52.5)$ & $<0.0001$ & 8.63 & $6.11-12.20$ \\
\hline Ampicillin-sulbactam & $82(7.2)$ & $21(11.7)$ & $\mathbf{0 . 0 3 6 7}$ & 1.71 & $1.03-2.84$ \\
\hline Vancomycin & $126(11.1)$ & $68(38)$ & $<0.0001$ & 4.91 & $3.45-7.00$ \\
\hline Ceph 1 & 387 (34.1) & $26(14.5)$ & $<0.0001$ & 0.33 & $0.21-0.51$ \\
\hline Ceph 2 & $81(7.1)$ & $12(6.7)$ & 0.84 & & \\
\hline Ceph $3^{e}$ & $108(9.5)$ & 44 (24.6) & 0.0011 & 3.10 & $2.09-4.60$ \\
\hline Macrolide & $37(3.3)$ & $17(9.5)$ & $<0.0001$ & 3.12 & $1.71-5.67$ \\
\hline Aminoglycosides & $187(16.5)$ & $79(44.1)$ & $<0.0001$ & 4.00 & $2.87-5.60$ \\
\hline Quinolones & $202(17.8)$ & 57 (31.8) & $<0.0001$ & 2.16 & $1.52-3.06$ \\
\hline
\end{tabular}

${ }^{a}$ Variables with $P$ values of $\leq 0.05$ are in boldface type.

${ }^{b} n=1,136$.

${ }^{c} n=179$.

${ }^{d}$ For cases, time at risk prior to $P$. aeruginosa isolation on clinical culture, and for controls, complete length of hospital stay.

${ }^{e}$ Excludes ceftazidime, which was not on formulary.

${ }^{f}$ Ceph 1 to 3, narrow-, expanded-, and broad-spectrum cephalosporins, respectively.

\section{DISCUSSION}

In this study we assessed risk factors for piperacillin-tazobactam-resistant $P$. aeruginosa and risk factors for piperacillintazobactam-susceptible $P$. aeruginosa. To our knowledge, this is the first study to describe the epidemiology of piperacillintazobactam-resistant $P$. aeruginosa. The identification of specific risk factors for emerging pathogens is important for a number of reasons. First, it allows the clinician a method of identifying patients at high risk of infection with resistant organisms, thus potentially leading to earlier diagnosis and better treatment. Second, the correct identification of specific risk factors is important in improving the understanding of the epidemiology, etiology, and pathogenesis of diseases caused by antibiotic-resistant organisms. Third, it identifies potentially modifiable risk factors that may lead to process-of-care changes (for example, changes in antibiotic utilization policies) that may decrease the emergence of antibiotic-resistant organisms among patients.

In this study, we found that piperacillin-tazobactam, imipenem, aminoglycosides, vancomycin, and broad-spectrum cephalosporins were the antibiotics statistically associated with the isolation of piperacillin-tazobactam-resistant $P$. aeruginosa. Time at risk (length of stay prior to the event), ICU stay, transfer from another medical institution, and the number of admissions in the previous year were also significant risk factors. Vancomycin and ampicillin-sulbactam were antibiotics statistically associated with piperacillin-tazobactam-susceptible $P$. aeruginosa.

The identification of piperacillin-tazobactam as a risk factor has biological plausibility in that the use of an antibiotic often leads to emergence of resistance to that particular antibiotic (9). In our study, 94 patients $(52.5 \%)$ with piperacillin-tazobactam-resistant $P$. aeruginosa had received piperacillin-tazobactam in the 14 days prior to positive culture.

There was a statistical association in the multivariable regression analysis of piperacillin-tazobactam-resistant $P$. aeruginosa with imipenem, aminoglycosides, vancomycin, and broadspectrum cephalosporins. Case-control studies of the nature done in this study do not necessarily identify causal associations; thus, these antibiotics may just be statistically significant as a result of the fact that patients who develop resistant pseudomonas likely receive a number of different antibiotics prior to their clinical culture positivity for $P$. aeruginosa. Hence, these results will need to be validated by other studies. However, these antibiotics were significantly associated in the multivariable model, and based on the fact that $47.5 \%$ of cases 
TABLE 2. Bivariate risk factors for the isolation of $P$. aeruginosa susceptible to piperacillin-tazobactam ${ }^{a}$

\begin{tabular}{|c|c|c|c|c|c|}
\hline \multirow{2}{*}{ Variable } & \multicolumn{2}{|c|}{ Value or no. $(\%)$ for: } & \multirow{2}{*}{$P$ value } & \multirow{2}{*}{ OR } & \multirow{2}{*}{$95 \%$ CI } \\
\hline & Controls $^{b}$ & Cases $^{c}$ & & & \\
\hline \multicolumn{6}{|l|}{ Demographics and comorbidities } \\
\hline Mean age (yr) & 49.7 & 55.3 & $<0.0001$ & 1.02 & 1.01-1.02 \\
\hline Male gender & $440(39.7)$ & $238(38.1)$ & 0.53 & & \\
\hline AIDS & $29(2.6)$ & $11(1.8)$ & 0.26 & & \\
\hline Cardiac disease & $126(11.4)$ & $107(17.2)$ & 0.0007 & 1.61 & $1.22-2.13$ \\
\hline Diabetes & $223(20.1)$ & $131(21.0)$ & 0.66 & & \\
\hline Malignancy & $100(9)$ & $63(10.1)$ & 0.46 & & \\
\hline Cerebrovascular accident & $65(5.9)$ & 95 (15.2) & $<0.0001$ & 2.88 & 2.07-4.02 \\
\hline Hepatic disease & $23(2.1)$ & $16(2.6)$ & 0.51 & & \\
\hline Renal disease & $27(2.4)$ & $10(1.6)$ & 0.25 & & \\
\hline Charlson comorbidity scale (mean) & 1.28 & 1.41 & 0.18 & & \\
\hline \multicolumn{6}{|l|}{ Variables related to hospitalization } \\
\hline Time at risk $(\text { days })^{d}$ & 9.54 & 12.98 & $<0.0001$ & 1.02 & $1.02-1.03$ \\
\hline ICU & $420(37.9)$ & $462(74)$ & $<0.0001$ & 4.68 & $3.77-5.81$ \\
\hline Surgery & $159(14.3)$ & $125(20)$ & 0.0021 & 1.50 & $1.16-1.94$ \\
\hline Number of admissions past year & 0.54 & 0.57 & 0.58 & & \\
\hline Transfer & $146(13.2)$ & $112(18.0)$ & 0.0072 & 1.44 & $1.10-1.89$ \\
\hline \multicolumn{6}{|l|}{ Antibiotics $^{f}$} \\
\hline Imipenem & $22(2)$ & $31(5)$ & 0.0005 & 2.58 & $1.48-4.50$ \\
\hline Piperacillin-tazobactam & $127(11.5)$ & $101(16.2)$ & 0.0051 & 1.49 & 1.13-1.98 \\
\hline Ampicillin-sulbactam & $77(6.9)$ & $101(16.2)$ & $<0.0001$ & 2.59 & $1.89-3.55$ \\
\hline Vancomycin & $118(10.6)$ & $122(19.6)$ & $<0.0001$ & 2.04 & $1.55-2.69$ \\
\hline Ceph 1 & $377(34)$ & $200(32.1)$ & 0.41 & & \\
\hline Ceph 2 & $80(7.2)$ & 74 (11.9) & 0.0011 & 1.73 & $1.24-2.41$ \\
\hline Ceph $3^{e}$ & $100(9)$ & $92(14.8)$ & 0.0003 & 1.74 & $1.29-2.36$ \\
\hline Macrolide & $37(3.3)$ & $26(4.2)$ & 0.38 & & \\
\hline Aminoglycosides & $180(16.2)$ & $105(16.8)$ & 0.75 & & \\
\hline Quinolones & $190(17.1)$ & $94(15.1)$ & 0.26 & & \\
\hline
\end{tabular}

${ }^{a}$ Variables with $P$ values of $\leq 0.05$ are in boldface type.

${ }^{b} n=1,109$.

${ }^{c} n=624$.

${ }^{d}$ For cases, time at risk prior to $P$. aeruginosa isolation on clinical culture, and for controls, complete length of hospital stay

${ }^{e}$ Excludes ceftazidime, which was not on formulary.

${ }^{f}$ Ceph 1 to 3 , narrow-, expanded-, and broad-spectrum cephalosporins, respectively.

did not receive piperacillin-tazobactam, there is a suggestion of independent risk associated with antibiotics other than piperacillin-tazobactam.

The class of broad-spectrum cephalosporins included the individual antibiotics ceftazidime and cefepime, which may induce AmpC production; this may explain their statistical

TABLE 3. Multivariable analysis of risk factors for the isolation of $P$. aeruginos $a^{a}$

\begin{tabular}{|c|c|c|c|}
\hline \multicolumn{2}{|c|}{$\begin{array}{c}\text { Piperacillin-tazobactam-resistant } \\
\text { P. aeruginosa }\end{array}$} & \multicolumn{2}{|c|}{$\begin{array}{c}\text { Piperacillin-tazobactam-susceptible } \\
\text { P. aeruginosa }\end{array}$} \\
\hline Variable & OR $(95 \% \mathrm{CI})$ & Variable & OR $(95 \% \mathrm{CI})$ \\
\hline $\begin{array}{l}\text { Piperacillin- } \\
\text { tazobactam }\end{array}$ & $6.82(4.56-10.21)$ & Vancomycin & $1.53(1.13-2.06)$ \\
\hline Imipenem & $2.42(1.19-4.94)$ & $\begin{array}{l}\text { Ampicillin- } \\
\text { sulbactam }\end{array}$ & $2.28(1.62-3.21)$ \\
\hline Aminoglycosides & $2.18(1.44-3.28)$ & Age & $1.01(1.01-1.02)$ \\
\hline Vancomycin & $1.87(1.21-2.89)$ & ICU stay & $3.76(3.00-4.71)$ \\
\hline Ceph $3^{c}$ & $2.38(1.45-3.88)$ & $\mathrm{CVA}^{b}$ & $1.84(1.29-2.63)$ \\
\hline $\begin{array}{l}\text { Admissions in } \\
\text { prior year }\end{array}$ & $1.36(1.20-1.54)$ & & \\
\hline ICU stay & $2.59(1.65-4.06)$ & & \\
\hline Transfer & $2.07(1.31-3.27)$ & & \\
\hline Time at risk & $1.02(1.01-1.03)$ & & \\
\hline
\end{tabular}

\footnotetext{
${ }^{a}$ Only statistically significant risk factors are shown in this table.

${ }^{b}$ Cerebrovascular accident.

${ }^{c}$ Ceph 3, broad-spectrum cephalosporin.
}

association (10). Although imipenem has been shown to be an inducer of $\mathrm{AmpC}$, its role in selecting for resistant strains in a hospital setting needs further investigation (14). Vancomycin was identified as a risk factor for both piperacillin-tazobactamsusceptible $P$. aeruginosa and piperacillin-tazobactam-resistant $P$. aeruginosa. This may just be a marker of complementary antibiotic selection. For example, physicians often prescribe vancomycin to add gram-positive coverage to their empirical gram-negative coverage. This association should have been removed by the collinearity diagnostics that were performed. However, vancomycin may be a true causal risk factor. One possible scenario is that its selective activity against grampositive bacteria may allow gram-negative bacteria, such as $P$. aeruginosa, to gain a selective advantage. This will need to be studied in more detail in future studies.

The identification of ICU stay and length of stay in the hospital as risk factors was not unexpected. They have been identified as risk factors in prior studies of antibiotic-resistant organisms $(4,5,7,8,11,13)$. Transfer from another hospital and number of admissions in a prior year are statistical associations that have excellent face validity; patients with either of these risk factors are more likely to have been exposed to additional antibiotics and to other patients with antibioticresistant organisms.

A limitation of the present study is that we were unable to 
assess the role of patient-to-patient transmission. It should be noted that cases and controls were selected from the same medical or surgical service and time of admission, so an attempt was made to control for transmission by a surrogate means. However, it is likely that not accounting for patient-topatient transmission would bias towards the null; i.e., among patients who acquire the organism from another patient, the importance of antibiotics as causal components may be diminished since the patient-to-patient transmission is the principal causal factor. In addition, for $P$. aeruginosa, it has been shown that cross-infection is a rare event (12). Another limitation is that we do not know the molecular mechanisms by which resistance was conferred in our study isolates.

An additional limitation is that since the control patients were not screened with active surveillance cultures for the presence of $P$. aeruginosa, it is possible that some of these patients might actually have represented case patients. However, this type of misclassification would make case and control patients more similar by including case patients in the controls and would serve only to underestimate the associations noted in this study.

In conclusion, this study suggests that piperacillin-tazobactam is a strong risk factor for the isolation of piperacillintazobactam-resistant $P$. aeruginosa. However, the study suggests that other antibiotics are also associated with the emergence of piperacillin-tazobactam resistance. The nosocomial isolation of piperacillin-tazobactam-resistant $P$. aeruginosa may be affected by multiple antibiotics. Limiting the use of piperacillin-tazobactam alone may not be sufficient to control the emergence of piperacillin-tazobactam-resistant $P$. aeruginosa.

\section{ACKNOWLEDGMENTS}

This work was supported by unrestricted grants from Wyeth-Ayerst and Merck and NIH grant K23AI01752-01A1.
We thank Colleen Reilly for database maintenance and data extraction.

\section{REFERENCES}

1. Centers for Disease Control and Prevention. 1996. National Nosocomial Infections Surveillance (NNIS) Report, October 1986-April 1996. Am. J. Infect. Control 24:380-388.

2. Chambers, H. F. 2000. Other B-lactam inhibitors, p. 291-299. In L. Mandell, J. E. Bennett, and R. Dolin (ed.), Principles and practice of infectious diseases. Churchill Livingstone, Philadelphia, Pa.

3. Deyo, R. A., D. C. Cherkin, and M. A. Ciol. 1992. Adapting a clinical comorbidity index for use with ICD-9-CM administrative databases. J. Clin. Epidemiol. 45:613-619.

4. Gaynes, R. P., and D. H. Culver. 1992. Resistance to imipenem among selected gram-negative bacilli in the United States. Infect. Control Hosp. Epidemiol. 13:10-14.

5. Harris, A. D., J. Castro, D. C. Sheppard, Y. Carmeli, and M. H. Samore. 1999. Risk factors for nosocomial candiduria due to Candida glabrata and Candida albicans. Clin. Infect. Dis. 29:926-928.

6. Harris, A. D., T. B. Karchmer, Y. Carmeli, and M. H. Samore. 2001. Methodological principles of case-control studies that analyzed risk factors for antibiotic resistance: a systematic review. Clin. Infect. Dis. 32:1055-1061.

7. Kaye, K. S., A. D. Harris, H. Gold, and Y. Carmeli. 2000. Risk factors for recovery of ampicillin-sulbactam-resistant Escherichia coli in hospitalized patients. Antimicrob. Agents Chemother. 44:1004-1009.

8. Lucas, G. M., N. Lechtzin, D. W. Puryear, L. L. Yau, C. W. Flexner, and R. D. Moore. 1998. Vancomycin-resistant and vancomycin-susceptible enterococcal bacteremia: comparison of clinical features and outcomes. Clin. Infect. Dis. 26:1127-1133.

9. McGowan, J. E., Jr. 1983. Antimicrobial resistance in hospital organisms and its relation to antibiotic use. Rev. Infect. Dis. 5:1033-1048.

10. Medeiros, A. A. 1997. Evolution and dissemination of beta-lactamases accelerated by generations of beta-lactam antibiotics. Clin. Infect. Dis. 24(Suppl. 1):S19-S45.

11. Morrison, A. J., Jr., and R. P. Wenzel. 1984. Epidemiology of infections due to Pseudomonas aeruginosa. Rev. Infect. Dis. 6(Suppl. 3):S627-S642.

12. Olson, B., R. A. Weinstein, C. Nathan, W. Chamberlin, and S. A. Kabins. 1984. Epidemiology of endemic Pseudomonas aeruginosa: why infection control efforts have failed. J. Infect. Dis. 150:808-816.

13. Ostrowsky, B. E., L. Venkataraman, E. M. D'Agata, H. S. Gold, P. C DeGirolami, and M. H. Samore. 1999. Vancomycin-resistant enterococci in intensive care units: high frequency of stool carriage during a non-outbreak period. Arch. Intern. Med. 159:1467-1472.

14. Sanders, C. C., P. A. Bradford, A. F. Ehrhardt, K. Bush, K. D. Young, T. A. Henderson, and W. E. Sanders. 1997. Penicillin-binding proteins and induction of AmpC beta-lactamase. Antimicrob. Agents Chemother. 41:20132015. 